\title{
, \\ Guided-Mode Resonance-Based Relative Humidity Sensing Employing a Planar Waveguide Structure
}

\author{
Petra Urbancova ${ }^{1, *(\mathbb{D}}$, Jakub Chylek ${ }^{2}$, Petr Hlubina ${ }^{2}\left(\mathbb{D}\right.$ and Dusan Pudis ${ }^{1}$ \\ 1 Department of Physics, Faculty of Electrical Engineering and Information Technology, University of Zilina, \\ Univerzitna 1, 01026 Zilina, Slovakia; pudis@fyzika.uniza.sk \\ 2 Department of Physics, Technical University Ostrava, 17. listopadu 2172/15, \\ 70800 Ostrava-Poruba, Czech Republic; jakub.chylek@vsb.cz (J.C.); petr.hlubina@vsb.cz (P.H.) \\ * Correspondence: urbancova@fyzika.uniza.sk
}

Received: 1 November 2020; Accepted: 25 November 2020; Published: 27 November 2020

\begin{abstract}
In this paper, we present a new type of guided-mode resonance (GMR)-based sensor that utilizes a planar waveguide structure (PWS). We employed a PWS with an asymmetric three-layer waveguide structure consisting of substrate/Au/photoresist. The ellipsometric characterization of the structure layers, the simulated reflectance spectra, and optical field distributions under GMR conditions showed that multiple waveguide modes can be excited in the PWS. These modes can be used for refractive index sensing, and the theoretical analysis of the designed PWS showed a sensitivity to the refractive index up to $6600 \mathrm{~nm}$ per refractive index unit (RIU) and a figure of merit (FOM) up to $224 \mathrm{RIU}^{-1}$. In response to these promising theoretical results, the PWS was used to measure the relative humidity (RH) of moist air with a sensitivity up to $0.141 \mathrm{~nm} / \% \mathrm{RH}$ and a FOM reaching $3.7 \times 10^{-3} \% \mathrm{RH}^{-1}$. The results demonstrate that this highly-sensitive and hysteresis-free sensor based on GMR has the potential to be used in a wide range of applications.
\end{abstract}

Keywords: guided-mode resonance; planar waveguide structure; humidity sensor; sensitivity; resonance wavelength; figure of merit

\section{Introduction}

Recent progress in controlling and guiding light using a planar waveguide has been strongly motivated by the acquisition of new optical devices with unique properties [1]. Guided mode resonance (GMR) is a very promising development that is based on the excitation of guided modes in a planar waveguide under phase-matching condition using a special coupling element such as a prism or diffraction grating [2]. Some of the guided light in the waveguide can leak and under certain phase-matching conditions these leaky modes can interfere with the non-coupled reflected or transmitted light waves. This interaction causes an ultra-narrow resonance dip or peak in the reflectance or transmittance spectra at a particular wavelength, angle, and polarization [3]. Due to its controllable linewidth, the GMR effect has become an important concept in optical devices such optical sensors, optical polarizers, band-pass and band-stop filters, electro-optical switches, and modulators [2-8].

The resonant condition of GMR is very sensitive to changes in the refractive index in the surrounding environment, hence GMR-based structures are very good candidates for high-performance refractive index (RI) sensors [2]. Changes in the refractive index of the surrounding medium are accompanied by a shift in wavelength [9], angle [10], intensity [11] or phase [12], which can be detected by the corresponding detection scheme. The RI describes the optical parameters of a material, which are directly related to its composition, thus RI detection is an important concept in relation to sensing in biology, chemistry, and other fields [13]. GMR-based biosensors play an important role because they are label-free and able to provide real-time detection [14,15]. 
The structural parameters and the optical properties of the GRM structure are crucial factors that determine the performance of the GMR-based sensor [3]. Some classic planar dielectric waveguides include a metal layer in the waveguide architecture, and these can provide unique optical properties [16]. Interesting results have been found for the symmetric metal-dielectric-metal (MDM) and dielectric-metal-dielectric (DMD) geometries and also their asymmetric types $[1,17,18]$. In particular, metal-cladding waveguide structures have shown very interesting results in high-performance sensing applications. Zhou et al. presented absorption-based sensing by employing a symmetrical metal-cladding waveguide, in which liquid analyte served as the guiding layer [19]. Wang et al. studied and fabricated an RI sensor with high sensitivity based on an asymmetric metal-cladding dielectric waveguide structure. In this waveguide structure, an analyte was used as the guiding layer and gold and air were used for the cladding layers $[13,20]$. Nesterenko et al. performed an analytical study of a low-loss waveguide structure and demonstrated the degree of waveguide mode loss by controlling the thickness of the spacer layer between the absorptive and waveguide layer [17]. Yang et al. theoretically studied a metal-dielectric multilayer structure with high sensitivity due to the coupling between the surface plasmon polariton mode and multi-order waveguide modes [21]. Our goal was to theoretically and experimentally investigate a metal-cladding waveguide structure with a photoresist guiding layer and an analyte (a moist air) as an infinite dielectric cladding. This waveguide geometry shows very promising sensing properties.

In this paper, we present a highly sensitive GMR-based sensor that utilizes a planar waveguide structure (PWS) consisting of substrate/Au/photoresist layers intended for the measurement of relative humidity of moist air. The reflectance was evaluated using a transfer matrix method and showed sensitivity to the refractive index up to $6600 \mathrm{~nm}$ per refractive index unit (RIU) and a figure of merit (FOM) up to $224 \mathrm{RIU}^{-1}$. Following the theoretical analysis, the PWS was used to measure the relative humidity $(\mathrm{RH})$ of moist air. The spectral reflectance measurements were performed for $s$ - and $p$ - polarized waves reflected from the PWS. To attain resonance excitation of guided modes, we employed a BK7 coupling prism and the attenuated total reflection. The GMR effect of the PWS caused by the excitation of guided mode, which is manifested in the form of a narrow, well-pronounced reflectance dip, had a high sensitivity to the change in relative humidity $(\mathrm{RH})$ of the moist air. The sensitivity to the $\mathrm{RH}$ and FOM were as high as $0.141 \mathrm{~nm} / \% \mathrm{RH}$ and $3.7 \times 10^{-3} \% \mathrm{RH}^{-1}$, respectively.

\section{Structure Design and Theoretical Background}

We proposed a PWS in the form of asymmetric three-layer waveguide as shown in Figure 1, in which the TE and TM guided modes can be excited if the resonant condition is fulfilled. A fused silica glass slide with a thickness of $t_{s}=0.7 \mathrm{~mm}$ and the refractive index $n_{s}$ was used as a substrate. The metal cladding layer, which serves as a coupling layer is formed by an Au film with a thickness of $t_{A u}=30 \mathrm{~nm}$ and the complex permittivity $\varepsilon_{A u}$. The waveguide layer consists of dielectric material AZ1505 photoresist with a thickness of $t_{p} \approx 200 \mathrm{~nm}$ and the refractive index $n_{d}$. The external medium is formed by moist air with a refractive index $n_{\text {air }}$.

The first aim was to determine the spectral reflectance and sensing properties of the PWS. In the case of multilayer structures, there are several approaches for obtaining the reflection and transmission coefficient of the electromagnetic field. One of these is a transfer matrix method (TMM), which is considered as one of the most powerful methods in contemporary theoretical physics [22]. The TMM considers the multilayer system of $m$ layers and the incident light in the form of the plane wave. Each layer of system $j,(j=1,2, \ldots, m)$ has the thickness $t_{j}$ and the optical properties are described by the wavelength-dependent complex refractive index $\widetilde{n}_{j}=n_{j}+\mathrm{i} k_{j}$ and complex dielectric function $\varepsilon_{j}=\varepsilon_{r j}+\mathrm{i} \varepsilon_{i j}=\widetilde{n}_{j}^{2}$, respectively. The transfer matrix of the whole multilayer system is called a scattering matrix (S) and is a product of the interface matrices $(\mathbf{I})$ and layer matrices $(\mathbf{L})[23,24]$. In the case of the proposed three-layer system (substrate/Au/photoresist) shown in Figure 1, the scattering matrix takes the form:

$$
\mathbf{S}=\mathbf{I}_{01} \mathbf{L}_{1} \mathbf{I}_{12} \mathbf{L}_{2} \mathbf{I}_{23} \mathbf{L}_{3} \mathbf{I}_{34}
$$


where $\mathbf{I}_{i j}$ is the matrix of refraction at the $i j$ interface and $\mathbf{L}_{j}$ is the phase matrix describing the propagation through $j$-th layer, and they are defined as:

$$
\begin{aligned}
\mathbf{I}_{i j} & =\frac{1}{t_{i j}}\left[\begin{array}{cc}
1 & r_{i j} \\
r_{i j} & 1
\end{array}\right], \\
\mathbf{L}_{j} & =\left[\begin{array}{cc}
e^{i \beta_{j}} & 0 \\
0 & e^{i \beta_{j}}
\end{array}\right] .
\end{aligned}
$$

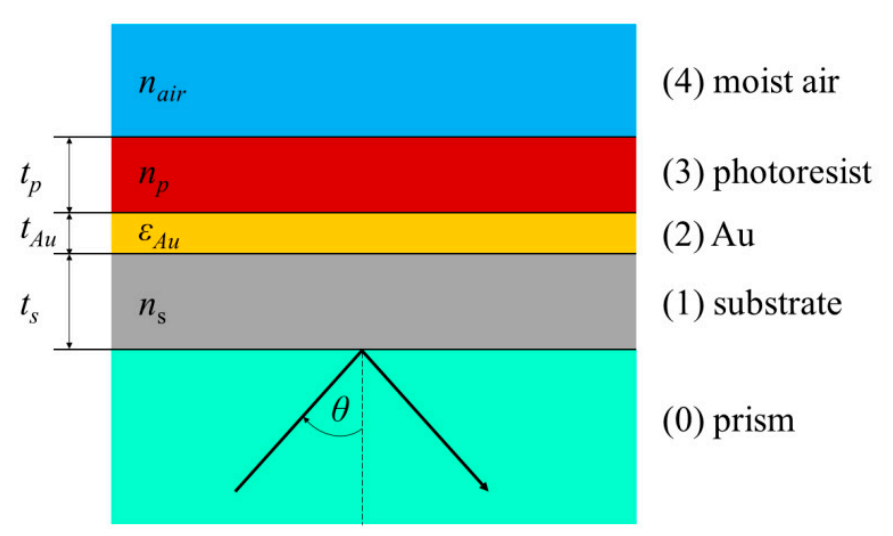

Figure 1. Scheme of the planar waveguide structure (PWS).

Coefficients $t_{i j}(\lambda)$ and $r_{i j}(\lambda)$ are Fresnel transmission and reflection coefficients at interface $i j$ and are different for $s$ - and $p$-polarized light wave, see [24], and $\beta_{j}$ is the phase thickness of the layer $j$ given as:

$$
\beta_{j}(\lambda)=\frac{2 \pi}{\lambda} n_{j}(\lambda) t_{j} \cos \theta_{j}=\frac{2 \pi}{\lambda} t_{j}\left[n_{j}^{2}(\lambda)-n_{0}^{2}(\lambda) \sin ^{2} \theta\right]^{\frac{1}{2}},
$$

where $\theta_{j}$ is the angle of refraction in the layer $j$. Reflectance $R_{s, p}(\lambda)$ for TE $(s)$ and TM $(p)$ waves of the multilayered structure can be expressed by the matrix elements of the scattering matrix $\mathbf{S}$ as:

$$
R_{s, p}(\lambda)=\left|\frac{S_{21}(\lambda)}{S_{11}(\lambda)}\right|_{s, p}^{2} .
$$

In the reflectance evaluations, the refractive index values of an external medium (moist air) were changed in the range of 1-1.005 with a step of 0.001 and the following dispersions of materials of the PWS were used. To model the response of the PWS, a BK7 coupling prism was included, whose dispersion is given by a Sellmeier formula:

$$
n(\lambda)=\sqrt{1+\frac{a \lambda^{2}}{\lambda^{2}-d}+\frac{b \lambda^{2}}{\lambda^{2}-e}+\frac{c \lambda^{2}}{\lambda^{2}-f}},
$$

where $\lambda$ is the wavelength in $\mu \mathrm{m}$ and the Sellmeier coefficients are as follows: $a=1.03961212$, $b=0.231792344, c=1.01046945, d=6.00069867 \times 10^{-3} \mu \mathrm{m}^{2}, e=2.00179144 \times 10^{-2} \mu \mathrm{m}^{2}$ and $f=1.03560653 \times 10^{2} \mu \mathrm{m}^{2}$. The dispersion of the substrate and AZ1505 photoresist was measured by a spectral ellipsometry method, when an ellipsometer RC2 (J. A. Woollam Co., Inc., Lincoln, NE, USA) was employed and the dispersion data fulfilled a Cauchy formula:

$$
n(\lambda)=\frac{a}{\lambda^{2}}+\frac{b}{\lambda}+c+d \lambda+e \lambda^{2}
$$


where $\lambda$ is the wavelength in $\mu \mathrm{m}$ and the Cauchy coefficients for the substrate are $a=0.01411 \mu \mathrm{m}^{2}$, $b=-0.04034 \mu \mathrm{m}, c=1.549, d=-0.03365 \mu \mathrm{m}^{-1}$ and $e=0.007592 \mu \mathrm{m}^{-2}$, and for the photoresist they are $a=0.03849 \mu \mathrm{m}^{2}, b=-0.1192 \mu \mathrm{m}, c=2.184, d=-0.1672 \mu \mathrm{m}^{-1}$ and $e=0.05318 \mu \mathrm{m}^{-2}$. The refractive indices of the substrate and AZ1505 photoresist given by Equation (7) are shown in Figure 2a. The dispersion of the Au layer obtained from ellipsometric measurements was described by the complex dielectric function given by the Drude-Lorentz model [25]:

$$
\varepsilon_{A u}(\lambda)=1-\frac{1}{\lambda_{p}^{2}\left(1 / \lambda^{2}+i / \gamma_{p} \lambda\right)}-\sum_{j=1}^{2} \frac{A_{j}}{\lambda_{j}^{2}\left(1 / \lambda^{2}-1 / \lambda_{j}^{2}\right)+i \lambda_{j}^{2} / \gamma_{j} \lambda},
$$

where $\lambda$ is the wavelength in $\mathrm{nm}$ and the parameters are specified in Table 1 . The real and imaginary part of the dielectric function of Au given by this model is shown in Figure 2b.

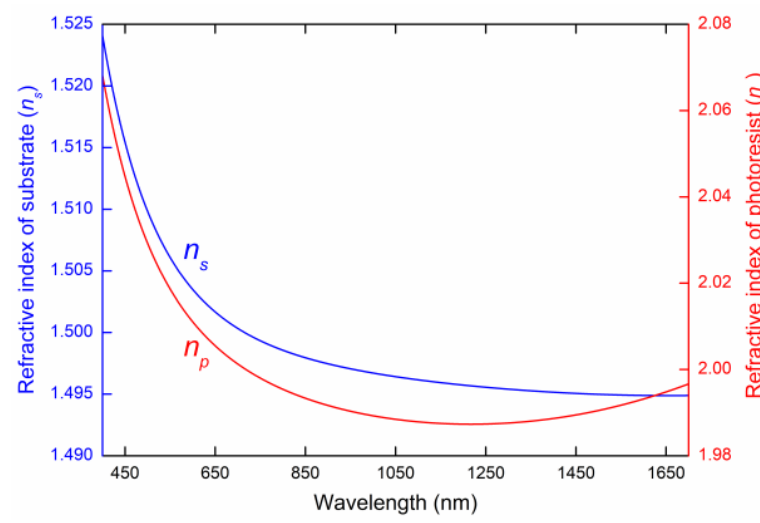

(a)

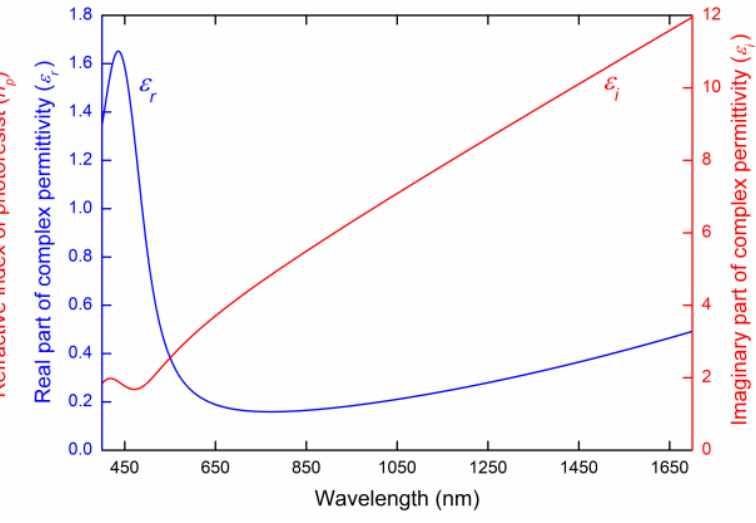

(b)

Figure 2. Dispersion of used materials. The refractive index of the substrate (blue line) and AZ1505 photoresist (red line) given by the Cauchy formula (a) and the real part (blue line) and imaginary part (red line) of the complex permittivity of the Au layer given by the Drude-Lorentz model (b).

Table 1. Parameters of the dielectric function of Au.

\begin{tabular}{cccccc}
\hline $\begin{array}{c}\text { Drude Term } \\
\text { Parameter }\end{array}$ & Value & $\begin{array}{c}\text { Oscillator 1 } \\
\text { Parameter }\end{array}$ & Value & $\begin{array}{c}\text { Oscillator 2 } \\
\text { Parameter }\end{array}$ & Value \\
\hline$\varepsilon_{\infty}$ & 1 & $A_{1}$ & 1.860 & $A_{2}$ & 3.439 \\
$\lambda_{p}(\mathrm{~nm})$ & 138.831 & $\lambda_{1}(\mathrm{~nm})$ & 419.828 & $\lambda_{2}(\mathrm{~nm})$ & 294.231 \\
$\gamma_{p}(\mathrm{~nm})$ & $21,687.402$ & $\gamma_{1}(\mathrm{~nm})$ & -39.047 & $\gamma_{2}(\mathrm{~nm})$ & -4192.008 \\
\hline
\end{tabular}

Figure 3 shows the theoretical spectral reflectances, $R_{s}(\lambda)$ and $R_{p}(\lambda)$ for given refractive index values of the analyte layer calculated for the angle of incidence $\theta=42.2^{\circ}$ with the following parameters: $t_{A u}=30 \mathrm{~nm}, t_{p}=200 \mathrm{~nm}$. The calculated reflectance spectra exhibit well pronounced dips with a constant width corresponding to guided modes $\mathrm{TE}_{1}, \mathrm{TE}_{0}$ (Figure $3 \mathrm{a}$ ) and $\mathrm{TM}_{1}$ (Figure $3 \mathrm{~b}$ ), respectively. The resonance wavelength shifts toward longer wavelengths with the increasing values of the refractive index of analyte. In Figure 3 we can see that the largest resonance wavelength shift regarding the change in $\mathrm{RI}$ is the $\mathrm{TE}_{0}$ mode, and on contrary, the mode with the smallest resonance wavelength shift is the $\mathrm{TE}_{1}$ mode. 


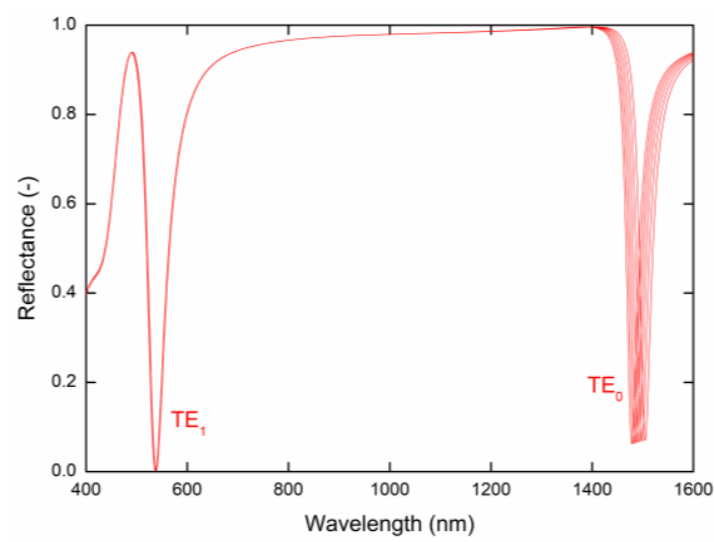

(a)

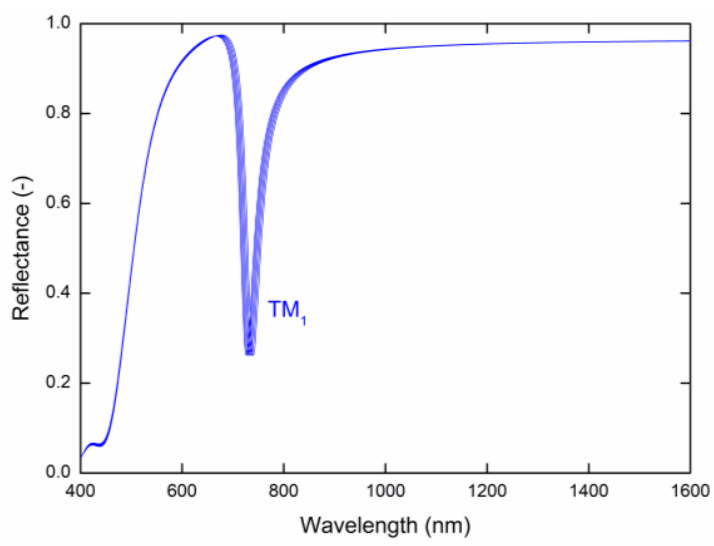

(b)

Figure 3. Theoretical spectral reflectances $R_{S}(\lambda)(\mathbf{a})$ and $R_{p}(\lambda)(\mathbf{b})$ for the PWS and analyte refractive index values in the range 1-1.005 with a step of 0.001 .

To describe the sensing properties of guided modes, it is necessary to determine the RI sensitivity $S_{n}$, which is defined as the change in the resonant wavelength $\delta \lambda_{r}$ with respect to the RI change $\delta n$ $\left(S_{n}=\delta \lambda_{r} / \delta n\right)$. The resonance wavelength of the $\mathrm{TM}_{1}$ and $\mathrm{TE}_{0}$ modes as a function of the analyte $\mathrm{RI}$ is shown in Figure $4 \mathrm{a}$ with a second-order polynomial fit, from which the RI sensitivity can be determined. The RI sensitivity $S_{n}$ shown in Figure $4 \mathrm{~b}$ is linearly dependent and changes approximately in the range $2300-3000 \mathrm{~nm} / \mathrm{RIU}$ for the $\mathrm{TM}_{1}$ mode, and 3900-6600 nm/RIU for the TE $\mathrm{E}_{0}$ mode, and the FOM, which is defined as a ratio of the sensitivity and the full width at half maximum (FWHM) of the $\operatorname{dip}\left(\mathrm{FOM}=S_{n} / \mathrm{FWHM}\right)$, achieves a value of $224 \mathrm{RIU}^{-1}$.

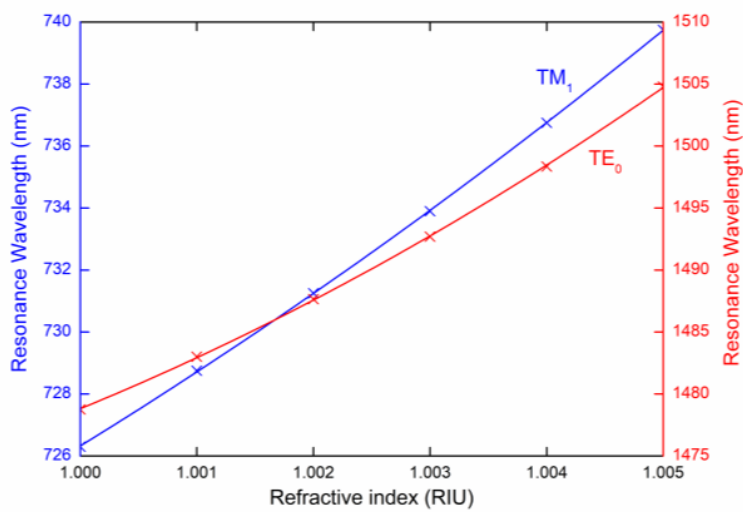

(a)

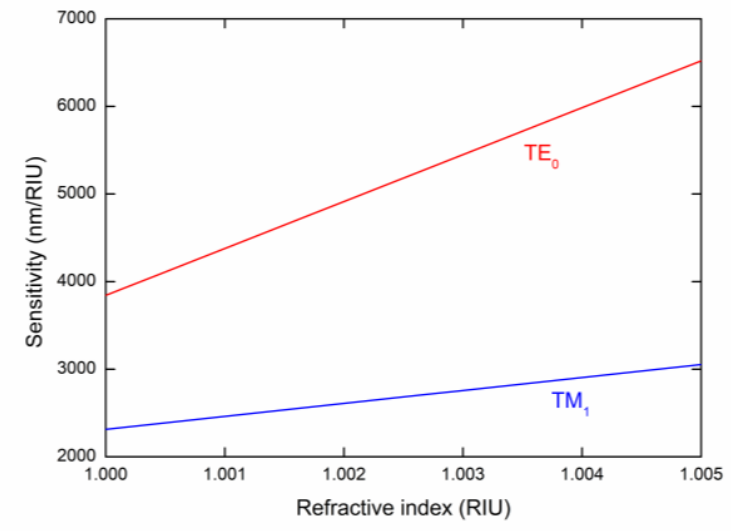

(b)

Figure 4. (a) Resonance wavelength and (b) sensitivity of the $\mathrm{TM}_{1}$ and $\mathrm{TE}_{0}$ modes as a function of the refractive index of the external medium.

In some cases, it is advantageous to consider the interference between the modes [26], which is attained when both the polarizer and analyzer are oriented $45^{\circ}$ with respect to the plane of incidence, and the corresponding reflectance $R_{45}(\lambda)$ is expressed as:

$$
R_{45}(\lambda)=\frac{1}{4}\left\{R_{s}(\lambda)+R_{p}(\lambda)+2 \sqrt{R_{s}(\lambda) R_{p}(\lambda)} \cos \left[\delta_{s p}(\lambda)\right]\right\}
$$

where $\delta_{s p}(\lambda)$ is the phase difference between the $s$ - and $p$-polarized waves. Figure 5a shows the theoretical spectral reflectance $R_{45}(\lambda)$ that correspond to the reflectances, $R_{p}(\lambda)$ and $R_{s}(\lambda)$ shown in Figure $3 a, b$. It is clear that the interference affects the depth of the dips. For the TM mode the depth is 
increased, while for the TE modes it is decreased. As demonstrated in Figure 5b, a slight shift in the resonance wavelength is present in comparison with Figure $4 a$, and the sensitivities are the same as those shown in Figure 4b.

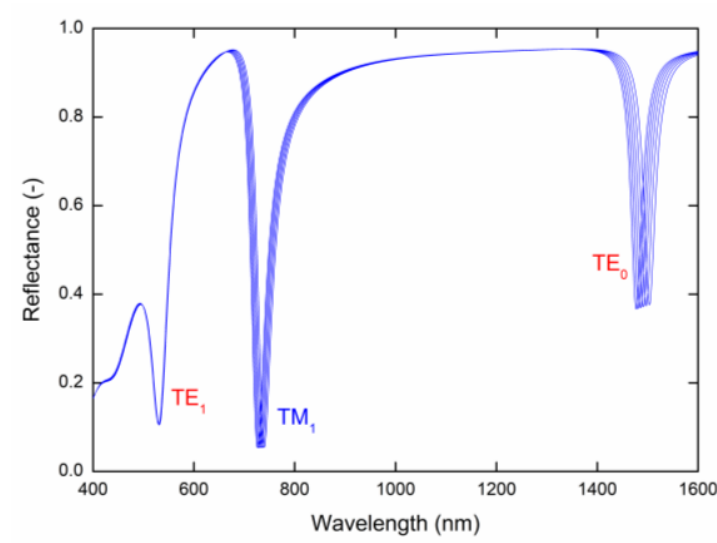

(a)

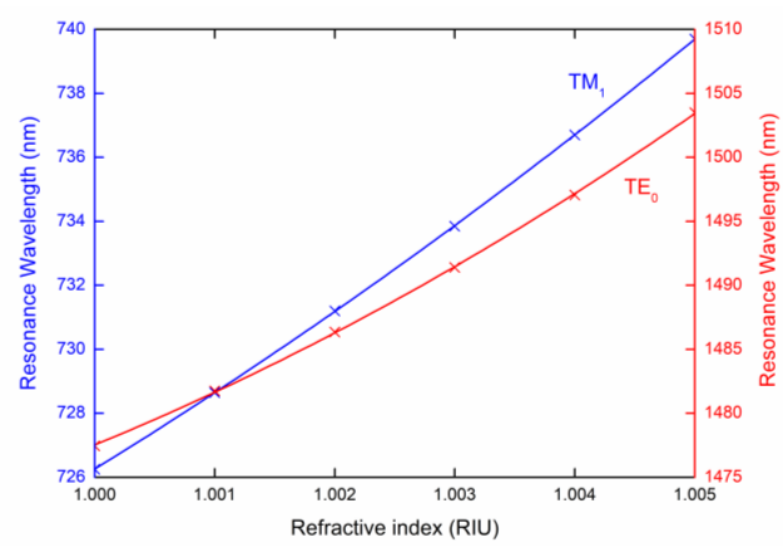

(b)

Figure 5. Theoretical spectral reflectances $R_{45}(\lambda)($ a) and resonance wavelength as a function of the refractive index of the external medium $(\mathbf{b})$.

A more detailed description of the GMR effect, that is, the optical field intensities $|E|^{2}$ in the PWS divided by $\left|E_{0}\right|^{2}$, where $E_{0}$ is the incident $s$-polarized or $p$-polarized electric field, are shown in Figure $6 \mathrm{a}, \mathrm{b}$, respectively. The normalized optical field intensities in the PWS shown in Figure 6a were calculated for the angle of incidence $\theta=42.2^{\circ}$ and a wavelength of $536.9 \mathrm{~nm}$ and correspond to the $\mathrm{TE}_{1}$ mode, and for the same angle of incidence and a wavelength of $1478.8 \mathrm{~nm}$ they correspond to the $\mathrm{TE}_{0}$ mode. While the $\mathrm{TE}_{1}$ mode only exhibits a 3-fold enhancement, the $\mathrm{TE}_{0}$ exhibits a 42-fold enhancement with very promising sensitivity. Figure $6 \mathrm{~b}$ shows the normalized optical field intensity in the PWS for the angle of incidence $\theta=42.2^{\circ}$ and a wavelength of $726.3 \mathrm{~nm}$ and corresponds to the $\mathrm{TM}_{1}$ mode, which exhibits a 47 -fold enhancement, however, with faster exponential decay in the analyte than for the $\mathrm{TE}_{0}$ mode. Thus, it is confirmed that theTE $\mathrm{E}_{0}$ mode has higher sensitivity than the $\mathrm{TM}_{1}$ mode for the considered analyte.

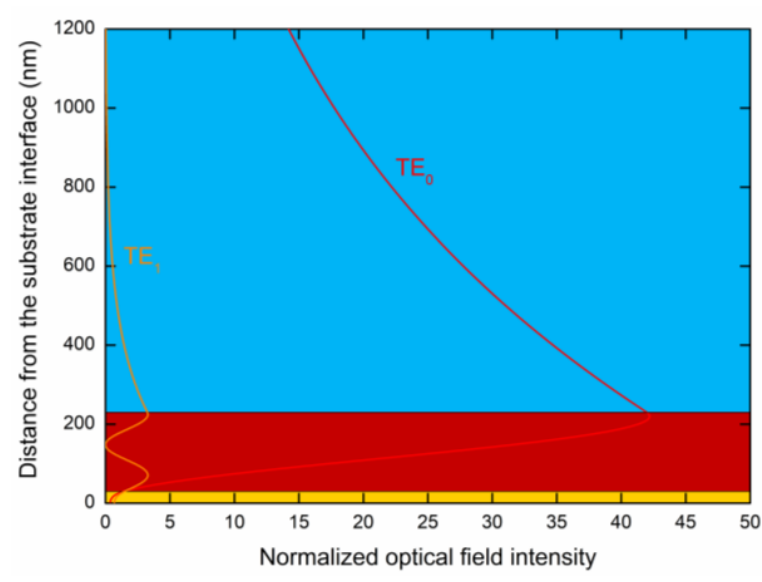

(a)

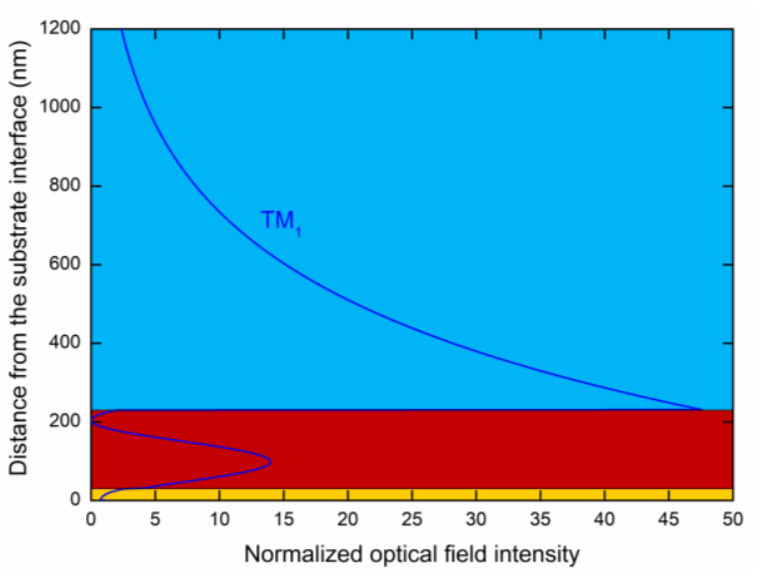

(b)

Figure 6. The normalized optical field intensity distribution in the PWS for the angle of incidence $\theta=42.2^{\circ}$ and for the wavelength (a) with $\lambda=536.9 \mathrm{~nm}$ exciting the $\mathrm{TE}_{1}$ mode and $\lambda=1478.8 \mathrm{~nm}$ exciting the $\mathrm{TE}_{0}$ mode, and (b) $\lambda=726.3 \mathrm{~nm}$ exciting the $\mathrm{TM}_{1}$ mode. 


\section{Experimental Setup}

The PWS structure under study consists of substrate/Au/photoresist layers. Fused silica glass slide serves as a substrate. The Au film was deposited on the chemically cleaned substrate, by a thermal evaporation process using a vacuum evaporator (K975X, Quorum Technologies Ltd., Laughton, East Sussex, UK). The thickness of the Au layer was measured using a film thickness monitor (10983, Quorum Technologies Ltd.) integrated in the evaporator during the evaporation process with a resolution of $0.1 \mathrm{~nm}$ thickness. The thin photoresist layer was deposited using an unadulterated positive photoresist AZ1505 using a spin-coating process (SPIN150, Semiconductor Production Systems Ldt., Coventry, UK). To remove the solvent from the photoresist layer, the samples were post-baked at $80^{\circ} \mathrm{C}$ for $2 \mathrm{~min}$. The ellipsometry measurements confirmed a homogeneous photoresist layer with a thickness $200 \pm 10 \mathrm{~nm}$ over the $1 \times 1 \mathrm{~cm}^{2}$ area.

The experimental setup shown in Figure 7 was used to measure the reflectance response of the PWS and the RH sensing ability in the VIS and NIR spectral ranges. We used a halogen lamp (HL-2000, Ocean Optics, Dunedin, FL, USA) as a white light source (WLS) with launching optics connected to an optical fiber (OF) with a collimating lens (CL). The collimated light beam of a $1 \mathrm{~mm}$ diameter was then polarized using a linear polarizer (P) (LPVIS050, Thorlabs, Newton, MA, USA) oriented $45^{\circ}$ with respect to the plane of incidence to generate both $p$ - and $s$-polarized components. The polarized light beam was coupled to the PWS using an equilateral prism made of BK7 glass (Ealing, Inc., South Natick, MA, USA) with index-matching fluid (Cargille, Cedar Grove, NJ, USA, $n_{D}=1.516$ ). The reflected light from PWS merges with a linear analyzer (A) (LPVIS050, Thorlabs) oriented $0^{\circ}, 90^{\circ}$ and $45^{\circ}$ with respect to the plane of incidence to generate the reflectances $R_{p}(\lambda), R_{s}(\lambda)$ and $R_{45}(\lambda)$ [26], respectively. The reflected light was launched into a spectrometer (USB4000, Ocean Optics) via a read optical fiber (ROF) (M15L02, Thorlabs) during the VIS measurements. In the NIR measurements, the reflected light was launched into an FT-NIR spectrometer (FT-NIR Rocket, ARCoptix S.A., Neuchatel, Switzerland) via a microscope objective and ROF (P400-2-VIS-NIR). The PWS was attached to a sensing chamber with a volume of approximately $22 \mathrm{~mL}$ via an $\mathrm{O}$ ring. To control the $\mathrm{RH}$ values in the sensing chamber, an electrical humidity and temperature sensor (HTS) (HTU21D, Arduino, Ivrea, Italy) connected to a controller board (Arduino UNO) was used. The adjusting system of the RH in the chamber comprises a humidifier and a two-line peristaltic pump (BT100M, Baoding Chuang Rui Precision Pump, Co., Ltd., Baoding, China). Adjusting the RH is described in detail in [26].

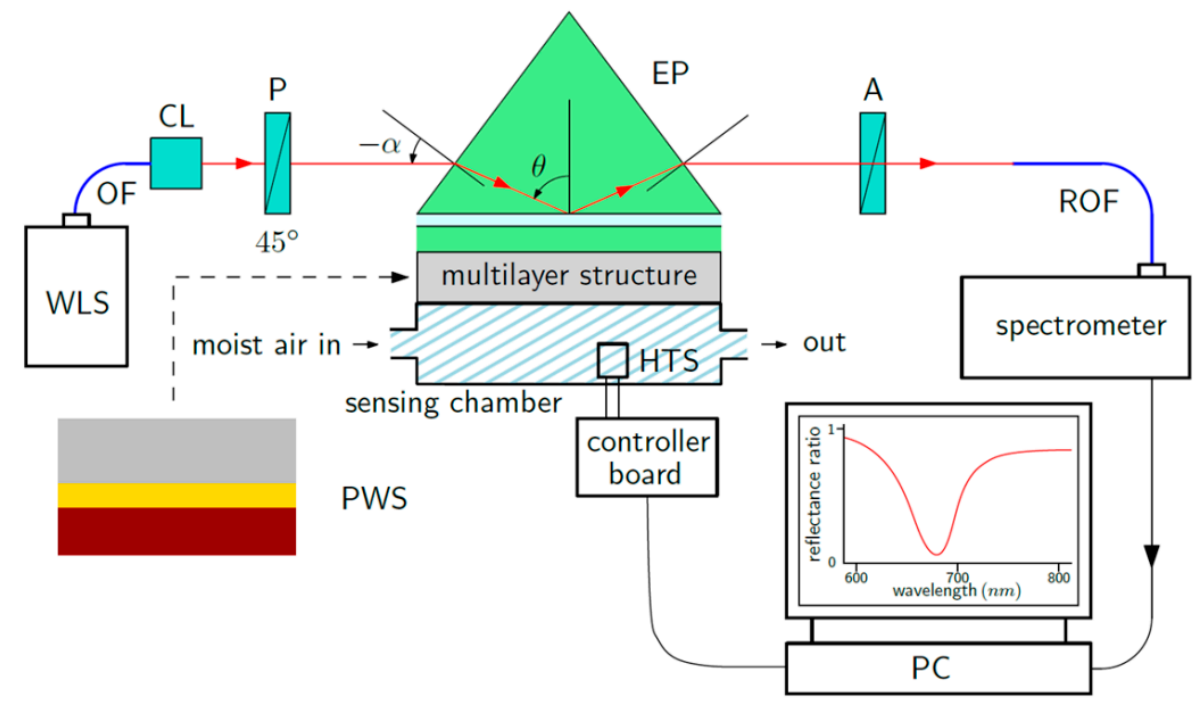

Figure 7. Scheme for the experimental setup. 


\section{Results and Discussion}

The GMR-based relative humidity measurements were performed at a temperature of $22.8^{\circ} \mathrm{C}$ (which was kept constant to avoid temperature cross-sensitivity), while the RH in the sensing chamber varied approximately in the range of $35 \% \mathrm{RH}$ to $85 \% \mathrm{RH}$. This was based on the spectral reflectance measurements for $s$ - and $p$-polarized waves reflected from the PWS. The reflectance ratios that induce the GMR effect as a function of the wavelength $\lambda$ were measured for two external angles of incidence (see Figure 7), $\alpha=16.6^{\circ}$ and $\alpha=20.6^{\circ}$, respectively. The resulting reflectance spectra are shown below and show the excitation of the guided modes accompanied by well-pronounced resonance dips in the spectra. The position of the dips that determines the resonance wavelength is red-shifted when the relative humidity of moist air increases. Figure 8 a shows the measured reflectance ratio $R_{\mathcal{S}}(\lambda) / R_{p}(\lambda)$ as a function of the wavelength for the external angle of incidence $\alpha=16.6^{\circ}$ and the relative humidity of the air in the range of $38.2 \% \mathrm{RH}$ to $80.1 \% \mathrm{RH}$. The figure shows the GMR for the $\mathrm{TE}_{1}$ mode accompanied by a well-pronounced dip. The resonance wavelength shift versus the RH change in the moist air is shown in Figure 8b with a second-order polynomial fit. The resonance wavelength was determined with a precision of $0.01 \mathrm{~nm}$ using a zero-crossing in the first derivative of the smoothed reflectance ratio. Figure 9a shows the GMR for the $\mathrm{TE}_{1}$ mode at the external angle of incidence $\alpha=20.6^{\circ}$. The relative humidity of the air was changed in the range of $36.1 \% \mathrm{RH}$ to $85.1 \% \mathrm{RH}$. Figure $9 \mathrm{~b}$ shows the resonance wavelength versus the RH change in moist air with a linear fit. The nonlinear to linear change in the resonance wavelength dependence on the $\mathrm{RH}$ can be attributed to the attenuated optical field in the surrounding medium. When the RH decreased, no hysteresis needed to be resolved in a quick response to the $\mathrm{RH}$ changes indicating that the surface optical field, as shown in Figure 6 is responsible for the sensing.

Figure 10a shows the wavelength dependence of the measured reflectance ratio $R_{45}(\lambda) / R_{S}(\lambda)$ responsible for the $\mathrm{TM}_{1}$ mode excitation, for the external angle of incidence $\alpha=16.6^{\circ}$ and the relative humidity of air in a range of $39.7 \%$ RH to $84.4 \%$ RH. In Figure $10 \mathrm{~b}$, shows the resonance wavelength shift towards longer wavelengths as the relative humidity of the air increases with a second-order polynomial fit of the measured data. Figure 11a shows the wavelength dependence of the measured reflectance ratio $R_{45}(\lambda) / R_{S}(\lambda)$ for the external angle of incidence $\alpha=20.6^{\circ}$ and the relative humidity of air in the range of $35.3 \% \mathrm{RH}$ to $85.7 \% \mathrm{RH}$. Figure $11 \mathrm{~b}$ shows the resonance wavelength shift again with the second-order polynomial fit of the measured data.

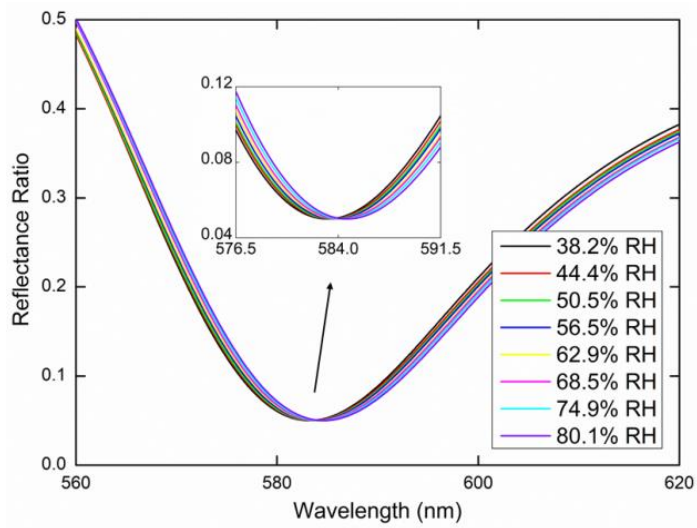

(a)

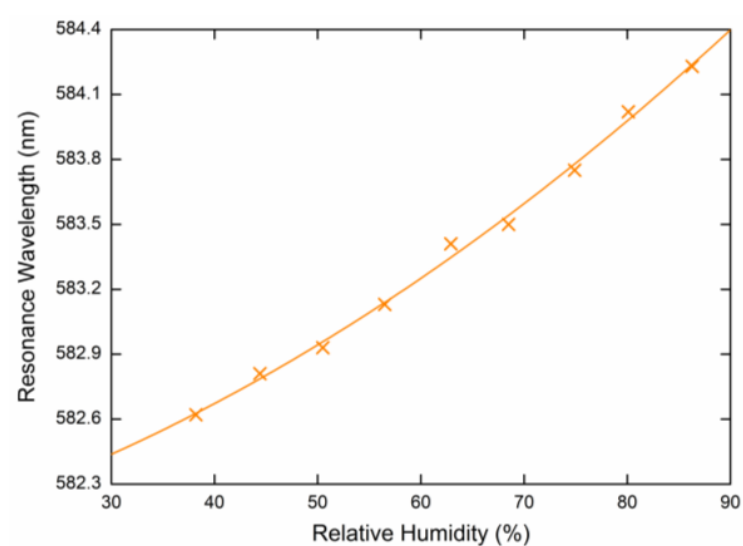

(b)

Figure 8. Measured spectral reflectance ratio $R_{\mathcal{S}}(\lambda) / R_{p}(\lambda)$ for the external angle of incidence $\alpha=16.6^{\circ}(\mathbf{a})$, the resonance wavelength as a function of the relative humidity $(\mathrm{RH})$ with a second-order polynomial fit (b). 


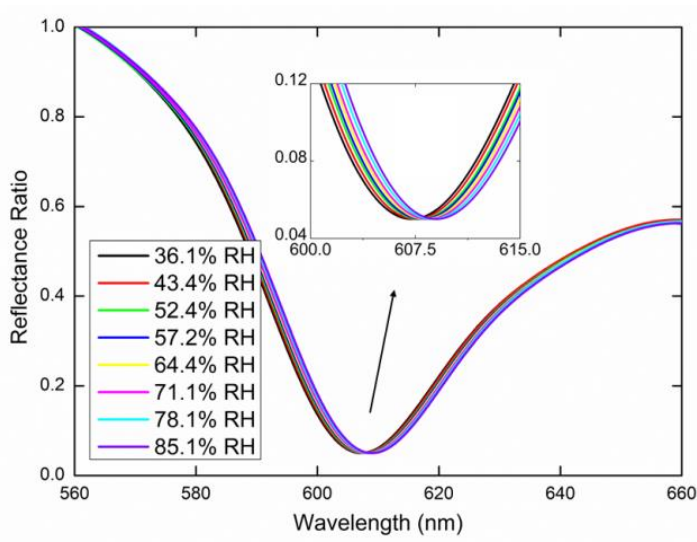

(a)

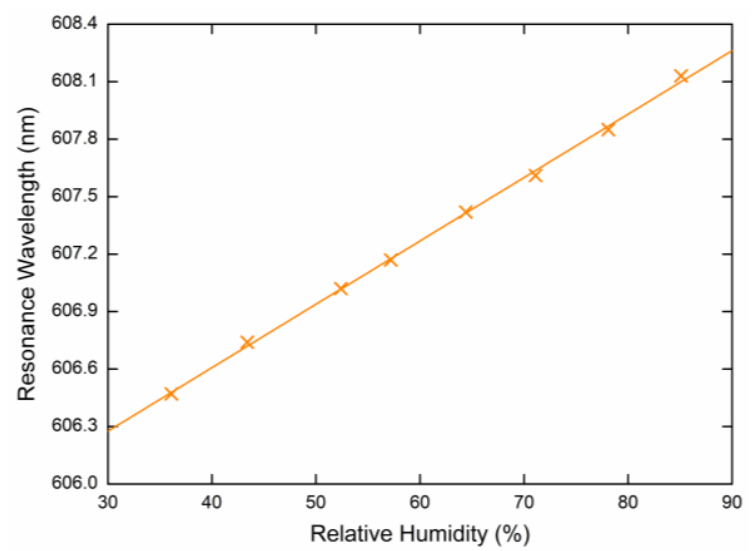

(b)

Figure 9. Measured spectral reflectance ratio $R_{\mathcal{S}}(\lambda) / R_{p}(\lambda)$ for the external angle of incidence $\alpha=20.6^{\circ}$ (a), the resonance wavelength as a function of the $\mathrm{RH}$ with a linear fit (b).

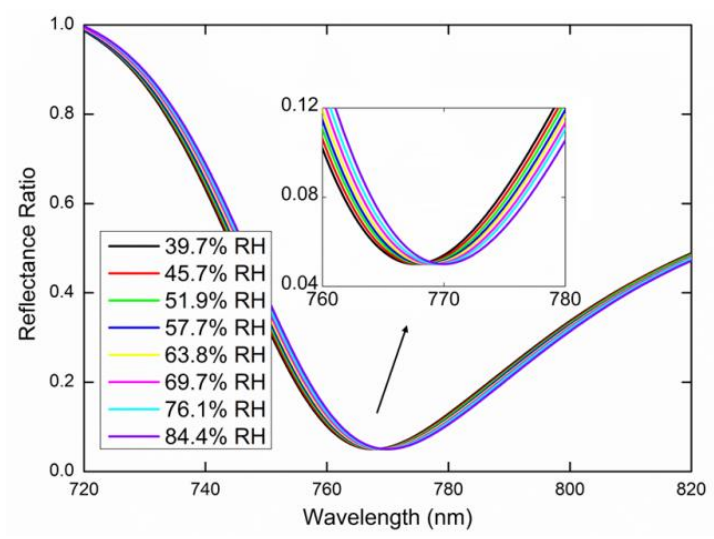

(a)

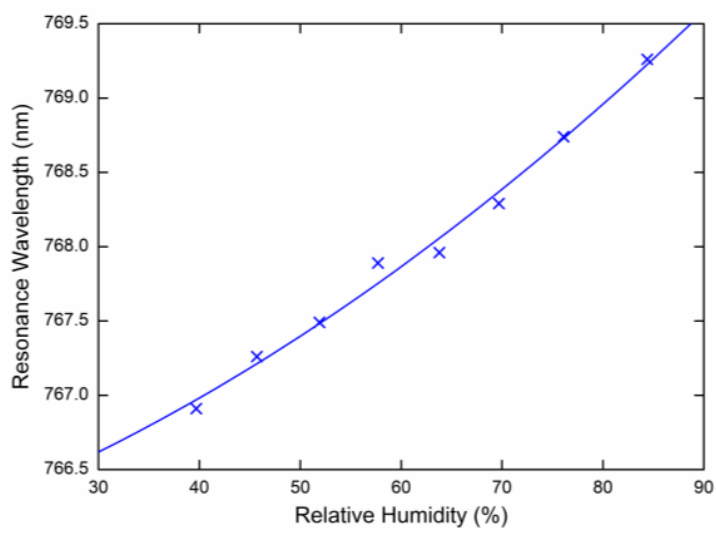

(b)

Figure 10. Measured spectral reflectance ratio $R_{45}(\lambda) / R_{s}(\lambda)$ for the external angle of incidence $\alpha=16.6^{\circ}(\mathbf{a})$, the resonance wavelength as a function of the $\mathrm{RH}$ with a second-order polynomial fit (b).

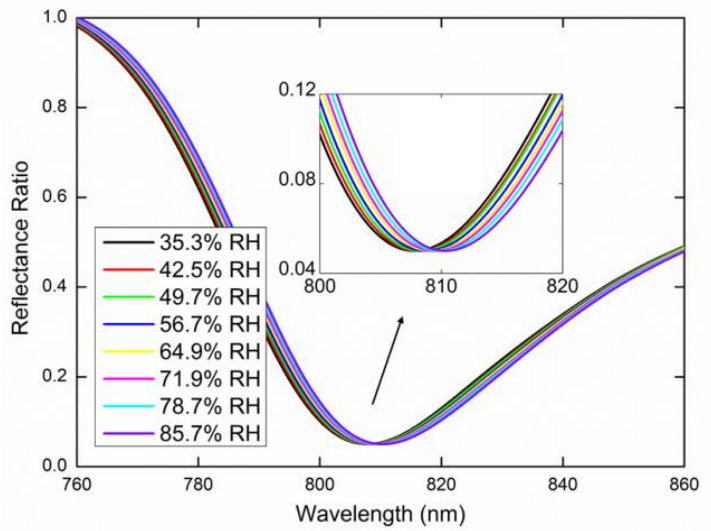

(a)

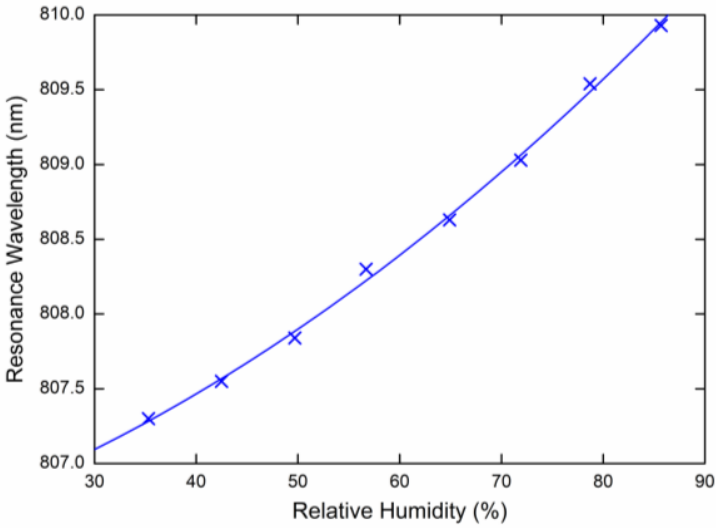

(b)

Figure 11. Measured spectral reflectance ratio $R_{45}(\lambda) / R_{S}(\lambda)$ for the external angle of incidence $\alpha=20.6^{\circ}$ (a), the resonance wavelength as a function of the $\mathrm{RH}$ with a second-order polynomial fit (b).

Lastly, the same measurements were performed for the $\mathrm{TE}_{0}$ mode excited by the s-polarized wave. Figure 12a or Figure 13a show the measured reflectance ratio $R_{s}(\lambda) / R_{p}(\lambda)$ for the external angle of incidence $\alpha=16.6^{\circ}$ and $\alpha=20.6^{\circ}$, respectively. Figure $12 \mathrm{~b}$ shows the resonance wavelength shift measured for the relative humidity ranging from $38.8 \% \mathrm{RH}$ to $84.6 \% \mathrm{RH}$ when the dependence was 
linearly fitted. Figure $13 \mathrm{~b}$ shows the resonance wavelength measured for the relative humidity in a range of $36 \% \mathrm{RH}$ to $85.8 \% \mathrm{RH}$ with a second-order polynomial fit.

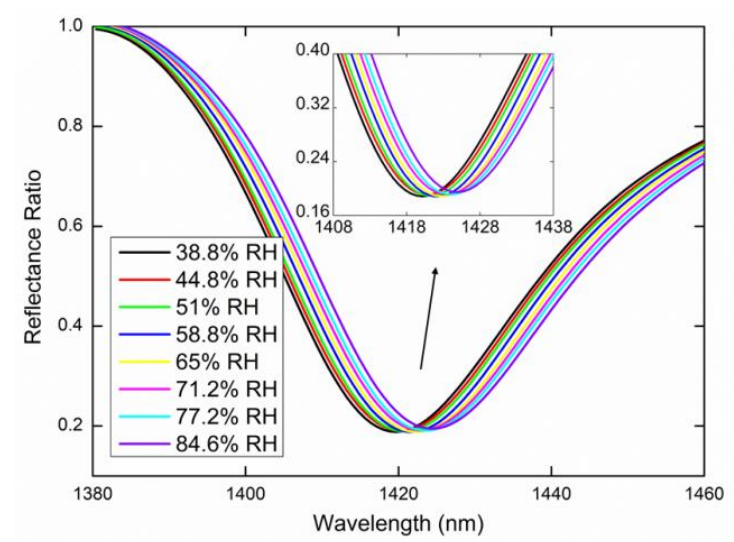

(a)

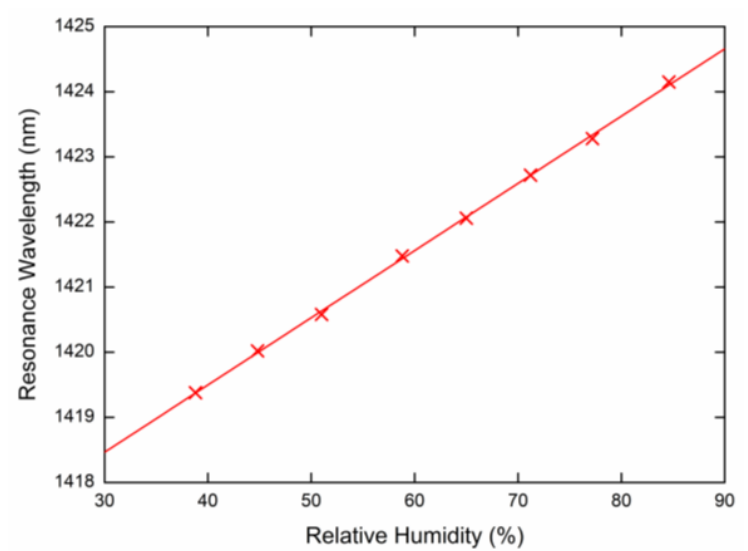

(b)

Figure 12. Measured spectral reflectance ratio $R_{S}(\lambda) / R_{p}(\lambda)$ for the external angle of incidence $\alpha=16.6^{\circ}(\mathbf{a})$, the resonance wavelength as a function of the $\mathrm{RH}$ with a linear fit $(\mathbf{b})$.

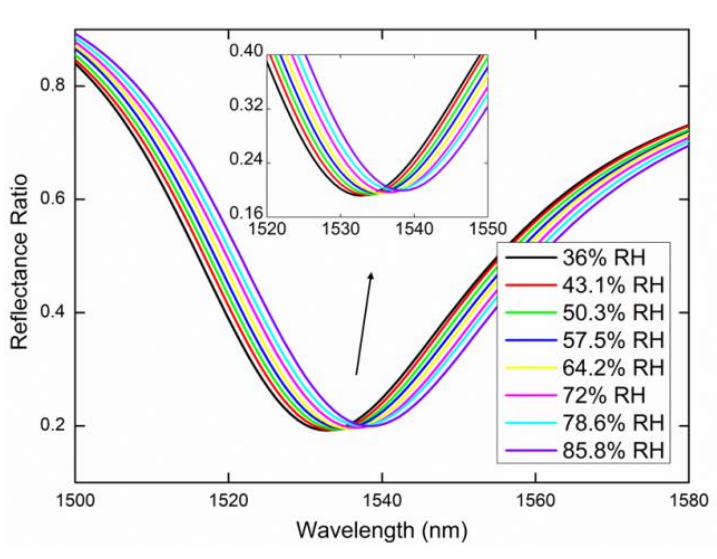

(a)

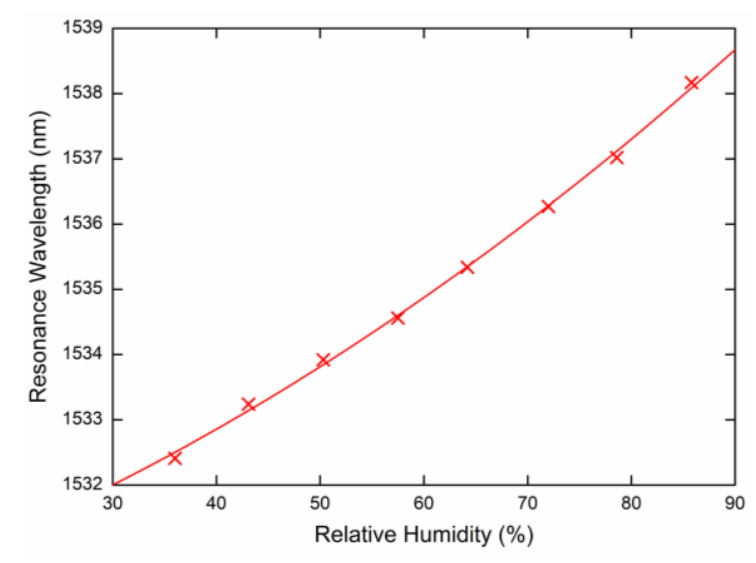

(b)

Figure 13. Measured spectral reflectance ratio $R_{\mathcal{S}}(\lambda) / R_{p}(\lambda)$ for the external angle of incidence $\alpha=20.6^{\circ}$ (a), the resonance wavelength as a function of the $\mathrm{RH}$ with a second-order polynomial fit (b).

To estimate the sensing properties of the PWS structure, it is necessary to determine the sensitivity to the relative humidity $S_{R H}$, which is defined as the change in the resonant wavelength $\delta \lambda_{r}$ with respect to the change in the relative humidity $\delta R H$ of moist air $\left(S_{R H}=\delta \lambda_{r} / \delta R H\right)$. From the measured shifts in the resonance wavelength and their respective linear polynomial dependence on the $\mathrm{RH}$, as shown in previous figures, we can determine the sensitivity $S_{R H}$ of the excited guided modes to the $\mathrm{RH}$. The achieved sensitivities $S_{R H}$ in a range of $35 \% \mathrm{RH}$ to $85 \% \mathrm{RH}$ are shown in Figure 14 .

For the $\mathrm{TE}_{1}$ mode, the sensitivity $S_{R H}$ exhibits a linear dependence on the $\mathrm{RH}$ in the range of 0.023 to $0.042 \mathrm{~nm} / \% \mathrm{RH}$ for the angle of incidence of $16.6^{\circ}$, as shown in Figure 14a. The sensitivity $S_{R H}$ of the same mode for the angle of incidence $\alpha=20.6^{\circ}$ is constant with a value of $0.033 \mathrm{~nm} / \% \mathrm{RH}$. The sensitivities $S_{R H}$ of the $\mathrm{TM}_{1}$ mode for the angles of incidence $\alpha=16.6^{\circ}$ and $\alpha=20.6^{\circ}$ are shown in Figure $14 \mathrm{~b}$ with values in the range of 0.036 to $0.063 \mathrm{~nm} / \% \mathrm{RH}$ and 0.037 to $0.068 \mathrm{~nm} / \% \mathrm{RH}$, respectively. Finally, the highest RH sensitivity exhibits the $\mathrm{TE}_{0}$ mode. For the angle of incidence $\alpha=16.6^{\circ}$, $S_{R H}$ reaches a constant value of $0.103 \mathrm{~nm} / \% \mathrm{RH}$ and for the angle of incidence $\alpha=20.6^{\circ}$, the dependence on the $\mathrm{RH}$ is linear and $S_{R H}$ varies in the range of 0.085 to $0.137 \mathrm{~nm} / \% \mathrm{RH}$, as shown in Figure 14a. 


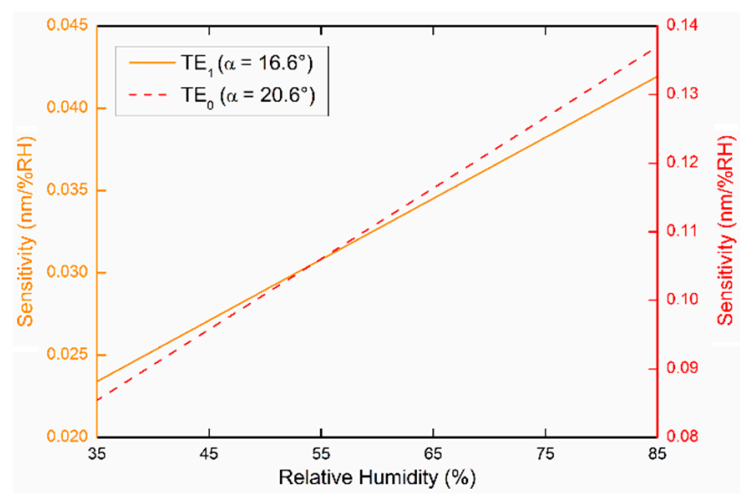

(a)

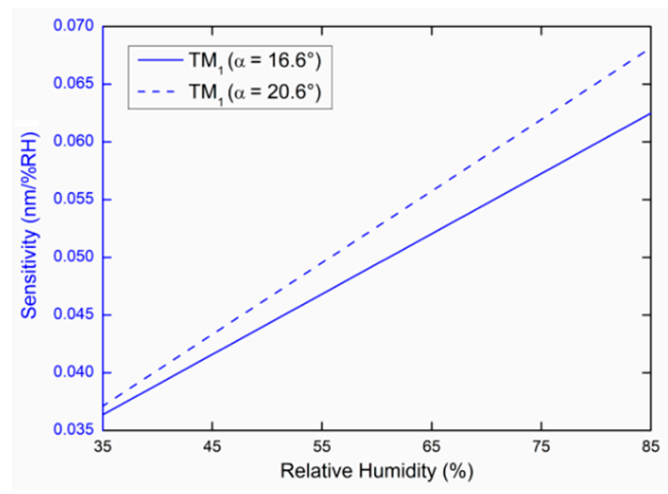

(b)

Figure 14. Sensitivities of guided modes measured at external angles of incidence $\alpha=16.6^{\circ}$ (a) and $\alpha=20.6^{\circ}(\mathbf{b})$.

The highest FOM, which is defined as a ratio of the sensitivity and the FWHM of the dip $\left(\mathrm{FOM}=S_{R H} / \mathrm{FWHM}\right)$, corresponds to the $\mathrm{TE}_{0}$ mode, which has the highest sensitivity $S_{R H}$ and the narrower resonance dip. The FOM attains a value of $3.1 \times 10^{-3} \% \mathrm{RH}^{-1}$ for the angle of incidence $\alpha=16.6^{\circ}$, as it is evident from Figure 12, and for the angle of incidence $\alpha=20.6^{\circ}$, the FOM is as high as $3.7 \times 10^{-3} \% \mathrm{RH}^{-1}$, as is shown in Figure 13 .

Table 2 summarizes various optical RH sensors with different principles and parameters, such as RH range and sensitivity, and the proposed RH sensor outperforms a number of them [26-28] in terms of sensitivity. These include sensors based on surface plasmon resonance [26], surface Bloch resonance [26], whispering gallery mode resonance [27] and guided mode resonance [28]. Some of the RH sensors [29-31] based on photonic crystal mode resonance [29] and lossy mode resonance [30,31] have higher sensitivity. However, to achieve a substantially higher sensitivity, fiber-optic RH sensors need to be implemented [32,33].

Table 2. Optical RH sensors with different parameters.

\begin{tabular}{ccccc}
\hline Material & Method & $\begin{array}{c}\text { RH } \\
\text { Range }\end{array}$ & $\begin{array}{c}\text { Sensitivity } \\
\text { (nm/\%RH) }\end{array}$ & Ref. \\
\hline plasmonic multilayer & surface plasmon wave resonance & $20-80 \%$ & 0.072 & {$[26]$} \\
dielectric multilayer & surface Bloch wave resonance & $22-80 \%$ & 0.065 & {$[26]$} \\
polymer coating & whispering gallery mode resonance & $0-60 \%$ & 0.013 & {$[27]$} \\
agarose gel & guided mode resonance & $20-80 \%$ & 0.150 & {$[28]$} \\
porous thin film & photonic crystal mode resonance & $11-84 \%$ & 0.296 & {$[29]$} \\
indium tin oxide & lossy mode resonance & $65-90 \%$ & 0.212 & {$[30]$} \\
copper oxide & lossy mode resonance & $30-90 \%$ & 0.636 & {$[31]$} \\
\hline
\end{tabular}

\section{Conclusions}

In this paper, we employed a three-layer PWS consisting of substrate/Au/photoresist to achieve highly sensitive and hysteresis-free measurement of the relative humidity of moist air. The measurement was based on resolving GMR for $s$ - and $p$-polarized waves reflected from the PWS. The GMR effect in the PWS was caused by the excitation of $\mathrm{TE}_{1}, \mathrm{TM}_{1}$ and $\mathrm{TE}_{0}$ guided modes, leading to narrow and well-pronounced dips in the reflectance spectra and a high sensitivity to the change in relative humidity of the moist air. We analyzed the sensitivity of all of the excited guided modes, and the sensitivity to the $\mathrm{RH}$ and FOM were as high as $0.141 \mathrm{~nm} / \% \mathrm{RH}$ and $188 \% \mathrm{RH}^{-1}$, respectively.

Finally, this simple sensing structure has a number of advantages, including a high sensitivity to the RI and FOM that achieved $6600 \mathrm{~nm} / \mathrm{RIU}$ and $224 \mathrm{RIU}^{-1}$, respectively. In addition, there is the potential to adjust the sensitivity as a constant by choosing a suitable angle of incidence. The use 
of the sensor can be extended to liquid analytes (working at different angles of incidence than for a moist air) where the polymer layer is substituted by a dielectric layer such as $\mathrm{SiO}_{2}$ [26]. Moreover, the sensor can be operated in aggressive environments because the layer acts as a protective overlayer for Au thin film, and the fiber-optic realization is possible because the sensor can be operated in the telecommunication window, near a wavelength of $1550 \mathrm{~nm}$.

Thus, the GMR-based sensor has the potential to be used in a wide range of applications.

Author Contributions: Conceptualization, P.U. and P.H.; methodology, P.U. and P.H.; software, J.C. and P.H.; validation, P.U., J.C., P.H. and D.P.; formal analysis, P.U. and P.H.; investigation, P.U., J.C. and P.H.; resources, P.U., P.H. and D.P.; data curation, P.U., J.C. and P.H.; writing-original draft preparation, P.U.; writing-review and editing, P.U. and P.H.; visualization, P.U. and P.H.; supervision, P.H. and D.P.; project administration, P.U.; funding acquisition, D.P. All authors have read and agreed to the published version of the manuscript.

Funding: This work was supported by the Slovak National Grant Agency under the project VEGA 1/0540/18 and the Slovak Research and Development Agency under the project No. APVV-16-0129 and APVV-19-0602. In addition, the research was supported by the ERDF/ESF project New Composite Materials for Environmental Applications (CZ.02.1.01/0.0/0.0/17_048/0007399), and by the student grant system through project SP2020/45.

Acknowledgments: The authors would like to thank Piere Koleják and Daniel Vala for performing ellipsometric measurements and data fitting.

Conflicts of Interest: The authors declare no conflict of interest.

\section{References}

1. Chen, L. Optical Devices Based on Symmetrical Metal Cladding Waveguides. In Optical Devices in Communication and Computation; Xi, P., Ed.; In Tech: Rijeka, Croatia, 2012; pp. 127-152.

2. Lan, G.; Zhang, S.; Zhang, H.; Zhu, Y.; Qing, L.; Li, D.; Nong, J.; Wang, W.; Chen, L.; Wei, W. High-performance refractive index sensor based on guided-mode resonance in all-dielectric nano-slit array. Phys. Lett. A 2019, 383, 1478-1482. [CrossRef]

3. Zhou, Y.; Wang, B.; Guo, Z.; Wu, X. Guided Mode Resonance Sensors with Optimized Figure of Merit. Nanomaterials 2019, 9, 837. [CrossRef] [PubMed]

4. Magnusson, R.; Wang, S.S. New principle for optical filters. Appl. Phys. Lett. 1992, 61, 1022. [CrossRef]

5. Sakat, E.; Vincent, G.; Ghenuche, P.; Bardou, N.; Dupuis, C.; Collin, S.; Pardo, F.; Haïdar, R.; Pelouard, J.-L. Free-standing guided-mode resonance band-pass filters: From 1D to 2D structures. Opt. Express 2012, 20, 13082-13090. [CrossRef]

6. Zheng, G.; Zou, X.; Xu, L.; Wang, J. Single layer narrow bandwidth angle-insensitive guided-mode resonance badstop filters. Optik 2017, 130, 19-23. [CrossRef]

7. Katchalski, T.; Levy-Yurista, G.; Friesem, A.A.; Martin, G.; Hierle, R.; Zyss, J. Ligh modulation with electro-optic polymer-based resonant grating waveguide structures. Opt. Express 2015, 13, 4645-4650. [CrossRef]

8. Forouzmand, A.; Mosallaei, H. Electro-optical Amplitude and Phase Modulators Based on Tunable Guided-Mode Resonance Effect. ACS Photonics 2019, 11, 2860-2869. [CrossRef]

9. Li, G.; Yang, J.; Zhang, Z.; Wen, K.; Tao, Y.; Han, Y.; Zhang, Z. Double Spectral Electromagnetically Induced Transparency Based on Double-Bar Dielectric Grating and Its Sensor Application. Appl. Sci. 2020, 10, 3033. [CrossRef]

10. Wei, X.; Weiss, S.M. Guided mode biosensor based on grating coupled porous silicon waveguide. Opt. Express 2011, 19, 11330-11339. [CrossRef]

11. Lin, Y.-C.; Hsieh, W.-H.; Chau, L.-K.; Chang, G.-E. Intensity-detection-based guided-mode-resonance optofluidic biosensing system for rapid, low-cost, label-free detection. Sens. Actuators B Chem. 2017, 250, 659-666. [CrossRef]

12. Sahoo, P.K.; Sarkar, S.; Joseph, J. High sensitivity guided-mode-resonance optical sensor employing phase detection. Sci. Rep. 2017, 7, 7607. [CrossRef] [PubMed]

13. Wang, X.; Wu, X.; Chen, Y.; Bai, X.; Pang, Z.; Yang, H.; Qi, Y.; Wen, X. Investigation of wide-range refractive index sensor based on asymmetric metal-cladding dielectric waveguide structure. AIP Adv. 2018, 8, 105029. [CrossRef] 
14. Triggs, G.J.; Wang, Y.; Peardon, C.P.; Fischer, M.; Evans, G.J.O.; Krauss, T.F. Chirped guided-mode resonance biosensors. Optica 2017, 4, 229-234. [CrossRef] [PubMed]

15. Shin, J.H.; Ok, G. Terahertz Guided Mode Resonance Sensing Platform Based on Freestanding Dielectric Materials: High Q-Factor and Tunable Spectrum. Appl. Sci. 2020, 10, 1013. [CrossRef]

16. Taya, S.; Elwasife, K. Guided modes in a metal-clad waveguide comprising a left-handed material as a guiding layer. IJRRAS 2012, 13, 294-305.

17. Nesterenko, D.V.; Hayashi, S.; Sekkat, Z. Extremely narrow resonances, giant sensitivity and field enhancement in low-loss waveguide sensors. J. Opt. 2016, 18, 065004. [CrossRef]

18. Anous, N.; Ramadan, T.; Abdallah, M.; Qaraqe, K.; Khalil, D. Planar broad-band and wide-range hybrid plasmonic IMI filters with induced transmission for visible light applications. Appl. Opt. 2017, 56, 8751-8758. [CrossRef]

19. Zhou, H.; Sang, Q.; Wang, X.; Chen, X. Symmetrical Metal Cladding Waveguide for Absorption Sensing and its Sensitivity Analysis. IEEE Photon. J. 2017, 9, 6800509. [CrossRef]

20. Wang, X.; Wu, X.; Zhu, J.; Pang, Z.; Yang, H.; Qi, Y. Theoretical Investigation of a Highly Sensitive Refractive-Index Sensor Based on $\mathrm{TM}_{0}$ Waveguide Mode Resonance Excited in an Asymmetric Metal-Cladding Dielectric Waveguide Structure. Sensors 2019, 19, 1187. [CrossRef]

21. Yang, L.; Wang, J.; Yang, L.; Hu, Z.-D.; Wu, X.; Zheng, G. Characteristics of multiple Fano resonances in waveguide-coupled surface plasmon resonance sensors based on waveguide theory. Sci. Rep. 2018, 8, 2560. [CrossRef]

22. Yeh, P. Optical Waves in Layered Media; John Wiley and Sons: Somerset, NJ, USA, 2005.

23. Nabok, A.; Tsargorodskaya, A. The method of total internal reflection ellipsometry for thin film characterization and sensing. Thin Solid Films 2008, 516, 8993-9001. [CrossRef]

24. Azzam, R.M.A.; Bashara, N.M. Ellipsometry and Polarized Light; North Holland Publishing Co.: Amsterdam, The Netherland, 1992; pp. 332-358.

25. Chlebus, R.; Chylek, J.; Ciprian, D.; Hlubina, P. Surface plasmon resonance based measurement of the dielectric function of a thin metal film. Sensors 2018, 18, 3693. [CrossRef] [PubMed]

26. Gryga, M.; Ciprian, D.; Hlubina, P. Bloch surface wave resonance based sensors as an alternative to surface plasmon resonance sensors. Sensors 2020, 20, 5119. [CrossRef] [PubMed]

27. Mehrabani, S.; Kwong, P.; Gupta, M.; Arman, A. Hybrid microcavity humidity sensor. Appl. Phys. Lett. 2013, 102, 241101. [CrossRef]

28. Lee, K.J.; Wawro, D.; Priambodo, P.S.; Magnusson, R. Agarose-Gel Based Guided-Mode Resonance Humidity Sensor. IEEE Sens. J. 2007, 7, 409-414. [CrossRef]

29. Peng, J.; Wang, W.; Qu, Y.; Sun, T.; Lv, D.; Dai, J.; Yang, M. Thin films based one-dimensional photonic crystal for humidity detection. Sens. Actuators A Phys. 2017, 263, 209-215. [CrossRef]

30. Fuentes, O.; Corres, J.M.; Matias, I.R.; Villar, I. Generation of Lossy Mode Resonances in Planar Waveguides Toward Development of Humidity Sensors. J. Lightwave Technol. 2019, 37, 2300-2306. [CrossRef]

31. Bohorquez, D.L.; Del Villar, I.; Corres, J.M.; Matias, I.R. Generation of lossy mode resonances in a broadband range with multilayer coated coverslips optimized for humidity sensing. Sens. Actuators B Chem. 2020, 325, 128795. [CrossRef]

32. Kolpakov, S.A.; Gordon, N.T.; Mou, C.; Zhou, K. Toward a new generation of photonic humidity sensors. Sensors 2014, 14, 3986-4013. [CrossRef]

33. Ascorbe, J.; Corres, J.M.; Arregu, F.J.; Matias, I.R. Recent developments in fiber optics humidity sensors. Sensors 2017, 17, 893. [CrossRef]

Publisher's Note: MDPI stays neutral with regard to jurisdictional claims in published maps and institutional affiliations.

(C) 2020 by the authors. Licensee MDPI, Basel, Switzerland. This article is an open access article distributed under the terms and conditions of the Creative Commons Attribution (CC BY) license (http://creativecommons.org/licenses/by/4.0/). 